\title{
Charge induced geometrical reorganization of DNA oligonucleotides studied by tandem mass spectrometry and ion mobility
}

Stefanie Ickert ${ }^{1,2}$, Johanna Hofmann ${ }^{3,4}$, Jens Riedel ${ }^{2}$, Sebastian Beck ${ }^{1}$, Kevin Pagel ${ }^{3,4}$, Michael W. Linscheid ${ }^{1 *}$

${ }^{1}$ Humboldt-Universitaet zu Berlin, Department of Chemistry, Brook-Taylor-Str. 2, 12489 Berlin

${ }^{2}$ Bundesanstalt für Materialforschung und -pruefung, Richard-Willstaetter-Str. 11, 12489 Berlin

${ }^{3}$ Fritz-Haber-Institut der Max-Planck-Gesellschaft, Faradayweg 4-6, 14195 Berlin

${ }^{4}$ Freie Universitaet Berlin, Takustr. 3, 14195 Berlin

*correspondence to: m.linscheid@chemie.hu-berlin.de

Keywords: Collision-induced dissociation (CID). Mass spectrometry (MS). Oligonucleotide (ON). Fragmentation. Ion mobility, Tandem-MS

\footnotetext{
Abstract

Mass spectrometry is applied as tool for the elucidation of molecular structures. This premises that gasphase structures reflect the original geometry of the analytes, while it requires a thorough understanding and investigation of the forces controlling and affecting the gas-phase structures. However, only little is known about conformational changes of oligonucleotides in the gas phase. In this study, a series of multiply charged DNA oligonucleotides $(n=15-40)$ has been subjected to a comprehensive tandem mass spectrometric study to unravel transitions between different ionic gas-phase structures. The nucleobase sequence and the chain length were varied to gain insights into their influence on the geometrical oligonucleotide organization. Altogether, 23 oligonucleotides were analyzed using collision-induced fragmentation. All sequences showed comparable correlation regarding the characteristic collision energy. This value that is also a measure for stability strongly correlates with the net charge density of the precursor ions. With decreasing charge of the oligonucleotides an increase in the fragmentation energy was observed. At a distinct charge density, a deviation from linearity was observed for all studied species, indicating a structural reorganization. To corroborate the proposed geometrical change, collisional cross sections of the oligonucleotides at different charge states were determined using ion mobility-mass spectrometry. The results clearly indicate that an increase in charge density and thus Coulomb repulsion results in the transition from a folded, compact form to elongated structures of the precursor ions. Our data show this structural transition to depend mainly on the charge density, whereas sequence and size do not have an influence.
} 


\section{Introduction}

The functionality of biomolecules is known to not only depend on the chemical composition but is hugely governed by the topological structure. Mass spectrometry (MS) is a widely applied technique to investigate molecules and soft ambient ionization techniques have led to insights into the native structure of biomolecules. In general, large biomolecules like oligonucleotides (ONs) and proteins are examined to gain a better understanding of biological processes based on information such as sequence or chemical modifications. Unlike proteins, DNA molecules larger than 100 base pairs can hardly be sequenced by MS, because of the complexity of generated fragment ion patterns. ${ }^{[1,2]}$

Thus, for DNA, MS analysis mainly focusses on studying molecular details like identification and location of modified bases or the identification of single nucleotide polymorphisms. ${ }^{[1]}$ For the latter, tandem MS is widely used and fragmentation of ONs has been comprehensively studied previously. ${ }^{[1]}$ In these MS/MS experiments the collision-induced dissociation (CID) of ONs was found to depend on sequence, base identity and charge state, ${ }^{[3-6]}$ while structural preconditions or general collisional stability were not addressed. ${ }^{[3-6]}$ However, other studies showed that the parent ion charge density has a significant influence on the observed fragments. ${ }^{[3]}$ This charge specific dissociation behavior led to the concept of the so called 'charge level', which is determined by the ratio of negative charges and the number of phosphate groups in an ON. ${ }^{[7]}$ Although it is known that large biomolecules of different charge levels have different geometries, the consequences for mass spectrometric behavior - here fragmentation reactions - and its use as a tool to study noncovalent interactions have yet to be specifically addressed. The charge level intrinsically includes considerations concerning the ions structure, since Coulomb repulsion, as driving force for unfolding, scales with the charge level. While DNA ONs are known to form coils under native aqueous conditions, a stretched linear form is energetically favored for gas-phase ions. ${ }^{[8]}$ In the electrospray ionization (ESI) process, multiply charged ions can be formed in energetically unfavored structures and may undergo conformational relaxation throughout a CID process. An insight into structural dynamics would therefore be highly desirable. $^{[9]}$

To further investigate how the fragmentation behavior of ONs correlates with their charge state and thus indirectly with the ion geometry, this study combines the results of three individual experiments to form a unified picture. First, a set of MS/MS experiments on multiply charged ON precursor ions was performed and the two observed main fragmentation channels of charged and neutral base loss were compared. Second, the fragmentation energy, i.e. the amount of collisional energy above which fragmentation occurs and that is proportional to the stability of the precursor ions, was investigated. 
These characteristic fragmentation energies of ONs were determined in dependence of the ON sequence, size and charge state. In a third set of experiments, the effective collisional cross sections (CCSs) of the precursor ions were determined by ion mobility-mass spectrometry (IM-MS) to elucidate the correlation of ion size to charge state.

\section{Experimental}

All oligonucleotides (Table 1) were purchased from Metabion International AG and analyzed at a final concentration of $10 \mu \mathrm{M}$ in water with $30 \%$ methanol and $1 \%$ triethylamine. Other chemicals were purchased from J.T. Baker and Carl Roth.

Negative ion ESI-MS spectra were obtained on a linear ion trap LTQ (Thermo Fisher Scientific, San Jose, CA, USA). The instrument parameters for all experiments were optimized according to the intensity of the seven-fold negatively charged oligonucleotide 5'-AAATTATAATTAGGC-3' and identified as: $100^{\circ} \mathrm{C}$ transfer capillary temperature and 30 arbitrary units as sheath gas flow. Sample solutions were directly infused at $2 \mu \mathrm{L} / \mathrm{min}$. The precursor ions were isolated for MS/MS experiments with an isolation width of $\Delta m / z 2$ and fragmented with a constant activation time of $30 \mathrm{~ms}$, while the collision energy was varied between $5 \%$ and $20 \%$ in steps of $0.1 \%$ until the abundance of the precursor ion signals decreased to $50 \%$ of its original value. This respective energy was then determined to be the characteristic fragmentation energy. In CID experiments, the applied collision energy was normalized (in \%) to compensate for the $m / z$ dependency of the fragmentation efficiency. ${ }^{[10]}$

IM-MS measurements were performed on a Synapt G2-S HDMS (Waters Corporation, Manchester, UK) that was modified with a linear drift cell, using a design reported previously. ${ }^{[11]}$ A nanoelectrospray source (nESI) was used to ionize typically $3 \mu \mathrm{L}$ of sample from platinum-palladium-coated borosilicate emitters prepared in-house. Parameters were: $0.9 \mathrm{kV}$ capillary voltage, $25 \mathrm{~V}$ sampling cone voltage, $25 \mathrm{~V}$ source offset voltage, $25^{\circ} \mathrm{C}$ source temperature, $2.0 \mathrm{~V}$ trap collision energy, $0 \mathrm{~V}$ transfer collision energy, $2 \mathrm{~mL} / \mathrm{min}$ trap gas flow. Ion mobility parameter were: 1.8 Torr helium IMS gas, $28^{\circ} \mathrm{C}$ IMS temperature, 4.6 V trap DC entrance voltage, 2.0 V trap DC bias voltage, $-9.0 \mathrm{~V}$ trap DC voltage, 6.6 V trap DC exit voltage, -20.0 V IMS DC entrance voltage, 45-85 V helium cell DC voltage, -40.0 V helium exit voltage, $60 \mathrm{~V}$ IMS bias voltage, $5.0 \mathrm{~V}$ transfer DC entrance voltage, $15.0 \mathrm{~V}$ transfer DC exit voltage, $222 \mathrm{~m} / \mathrm{s}$ trap wave velocity, $6.0 \mathrm{~V}$ trap wave height voltage, $400 \mathrm{~m} / \mathrm{s}$ transfer wave velocity, 10.0 V transfer wave height voltage. IM-MS spectra were recorded in the negative ion mode and drift times were converted to collision cross sections (CCSs) by using the Mason-Schamp equation, as described elsewhere. ${ }^{\text {[11-13] }}$ 


\section{Results and Discussion}

23 single strand DNA ONs were investigated that ranged in their size from 15 to 40 nucleotides and differed in their sequence (Supplementary Material, Table S1). In a first set of experiments, tandem mass spectra were generated of all observed charge states of the ONs and base losses were observed as most abundant fragmentation pathways. In general, two types of base losses were commonly observed: neutral and negative base loss. As previously shown, fragment formation and charge were strongly interdependent. ${ }^{[3-5,14]}$ Comparable trends in fragment ion distributions were observed for all ONs and, therefore, only the results for the 15-mers are exemplarily discussed here (Fig. 1). Neutral base loss was apparent with strong abundance in the MS/MS spectra of the three- to five-fold negatively charged ions (Fig. 1b). In case of the six-fold negatively charged parent ions, the first onset of negative base loss was observed. At a charge level of 0.5, meaning every second phosphate on average carries a negative charge, neutral base loss was still the most abundant signal, but the negative base loss signal had increased to $\sim 80 \%$ relative abundance in the MS/MS spectrum. With further increase of the precursor ion charge, the neutral base loss disappeared and the negative base loss increased in intensity (Fig. 1b). With high charge levels of the precursor ion the intramolecular Coulomb repulsions increase and thus the precoursors are more likely to release a charged rather that a neutral base in order to decrease the charges on the remaining strand. To investigate the underlying energetics, the fragmentation energies for

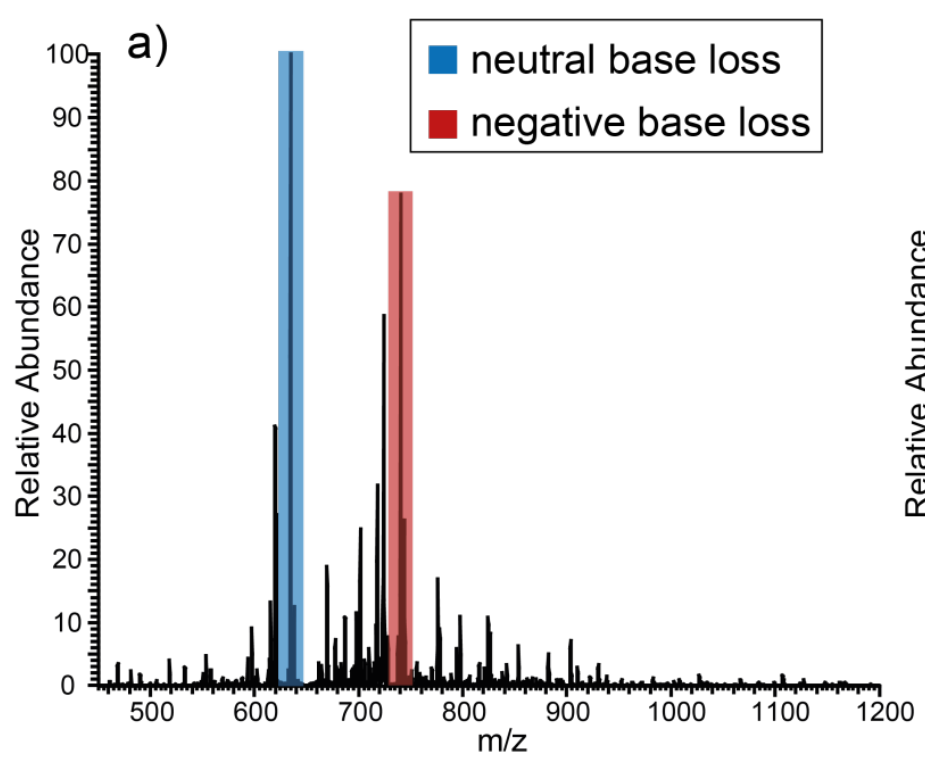

b)

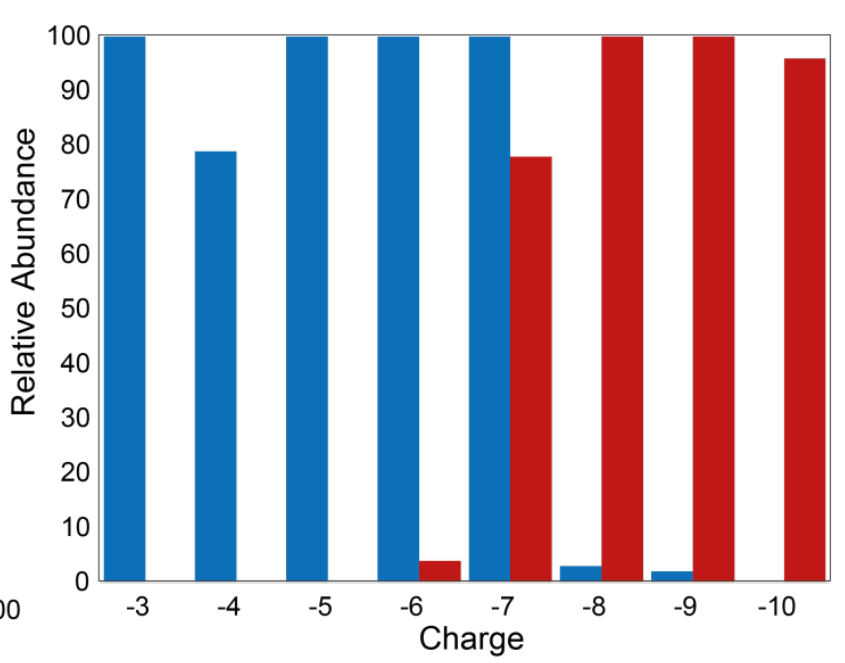

Figure 1. ESI-MS/MS spectra of the 15-mer ON 5'-AAATAAGGCCGCTAT-3'. a) MS/MS spectrum of the sevenfold negatively charged ion. The main fragmentation channels were neutral adenine base loss $[\mathrm{M}-7 \mathrm{H}-\mathrm{A}]^{7-}$ at $\mathrm{m} / \mathrm{z} 634.7$ (blue) and the negative base loss [M-6H-A $]^{6-}$ at $\mathrm{m} / \mathrm{z} 740.6$ (red), with relative signal intensities of 100 $\%$ and $79 \%$, respectively. b) Relative abundances of the neutral (blue) and the negatively charged (red) base losses. Fragmentation spectra for the three- to tenfold negatively charged precursor ions were recorded. 
the individual oligonucleotide charge states were determined. This allowed to correlate precursor ion stability to length, sequence and charge.

Ions, corresponding to each signal of the charge series, were isolated and the collision energy (CE) was increased in $0.1 \%$ steps until the characteristic fragmentation energy value was found. As an example two fragment spectra of the seven-fold negatively charged precursor ion of the oligonucleotide 5'-AAATTATAATTAGGC-3' with $\mathrm{m} / \mathrm{z} 656.1$ are shown at only slightly different collision energies (Fig. 2). Evidently, up to a CE of $12.5 \%$ no fragment ions were observed. Once the collision energy was increased to $12.6 \%$ all precursor ions fragmented quantitatively, resulting in complete disappearance of the precursor ion signal. Further increase of the CE beyond this energy resulted in no notable change in the spectra. These fragmentation energies were determined for at least eight charge states of each of the 15-, 24-, 32-, and 40-mers. In Fig. 3a a comprehensive correlation between CE and the charge states is given. To obtain reliable results, these collision energy values were determined as the averages of five independent measurements. The activation time of $30 \mathrm{~ms}$ in the ion trap collision cell ensured a comprehensive distribution of the energy transferred to the analyte ions via inelastic scattering. Hence, the initially formed conformers can take up energy until a certain thermodynamically controlled threshold is reached, at which the ions enter a dissociative exit channel. The potential energy of this threshold can be regarded as a measure for the overall stability of the precursor ion. This stability can increase by a conformational stabilization via charge delocalization over the whole molecule.
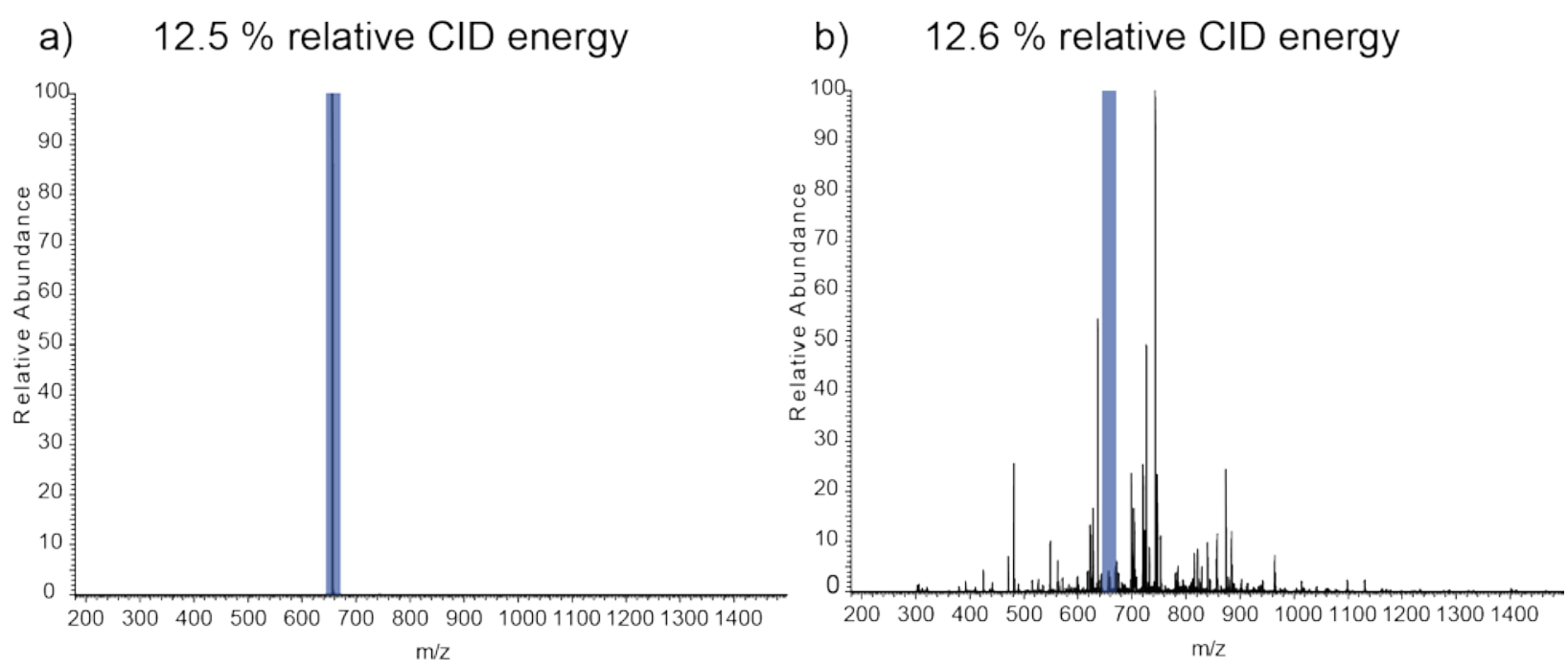

Figure 2. ESI-MS/MS spectra of the sevenfold negatively charged 15-mer ON 5'-AAATTATAATTAGGC-3'. a) MS/MS spectrum of the isolated precursor ion $\mathrm{m} / \mathrm{z} 656.1$ after CID activation at a relative CE of $12.5 \%$. b) MS/MS spectrum of the isolated precursor ion $\mathrm{m} / \mathrm{z} 656.1$ after CID activation at a CE of $12.6 \%$. The precursor ion $\mathrm{m} / \mathrm{z} 656.1$ is marked in blue. 

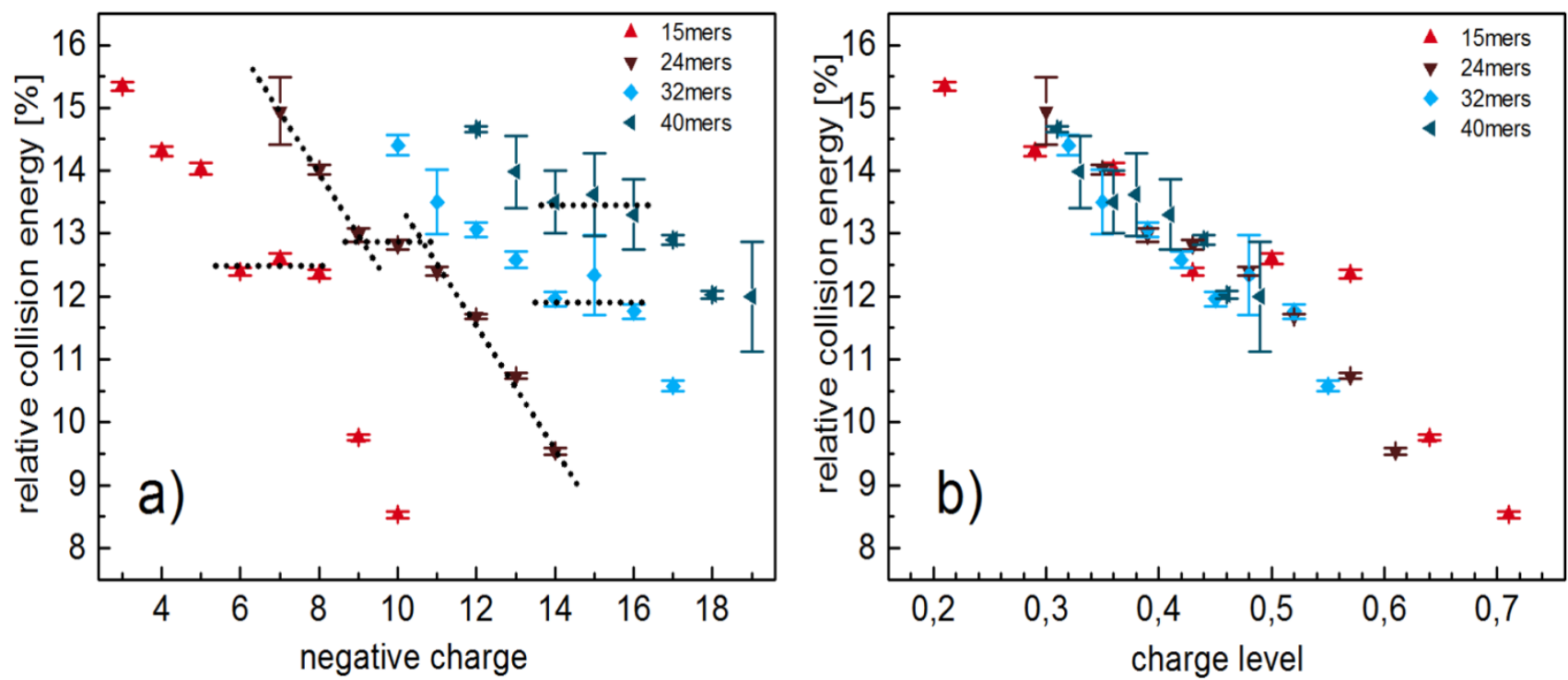

Figure 3. Correlation between the fragmentation energy and the charge state of ONs. a) The characteristic fragmentation energy of each ON size is plotted against the charge state. With increasing number of charges, less $\mathrm{CE}$ is needed to obtain fragmentation. Error bars represent the standard deviation of five individual measurements. Dotted lines serve as a guide to the eye to highlight the linear regions (for the 24-mer) as well as the inflexion zones. b) For better comparison of the different ON sizes, the fragmentation energies are plotted against the charge level (amount of negative charge per phosphate group). Independent of sequence and size of the ONs a similar fragmentation behavior was observed.

The distributions of fragmentation energies obtained for different oligonucleotide sizes in Fig. 3a revealed that all curves follow a common trend. The energies shift towards lower values with increasing numbers of charges on the precursor ions. Around the highest and lowest observable charge states this decrease is linear with a comparable slope and intercept for each ON chain length. For intermediate charge states, an inflexion zone appears.

An increasing number of charges naturally leads to increased Coulomb repulsion, which in turn leads to destabilization of $\mathrm{ON}$ ions. Within longer ONs, charge redistribution is more effective, so the amount of this destabilization energy per additional charge becomes less. This is reflected in the fragmentation energies, while the 15-mers fragmented between $8.5 \%$ and $15.4 \% \mathrm{CE}$, the 40 -mers fragmented in a range from $11.0 \%$ to $14.7 \%$ CE. The relevant factors in ON CID seem to be the net charge and the size, in other words the net charge per nucleobase or the charge level. This is also shown in a plot of the fragmentation energies against the charge level (Fig. 3b), where all investigated ON sizes show a common behaviour. Mechanistically, at the inflexion zone the deviation from linearity means that further charging of the parent ion does not lead to further destabilization. Because chain length and the charge state are defined, the repulsion of charges has to be reduced via a conformational change. The inflexion regions for all ONs are close to a charge level of 0.5 , more precisely for the 15 -mers 0.49 , for 
the 24-mers 0.43 , for the 32-mers 0.49 and for the 40 -mers 0.39 . This surprisingly coincides with the charge level at which neutral base loss disappeared in favor of charged base loss in the tandem MS experiments. In addition, it is shown that the characteristic fragmentation energy at a given charge level is not dependent on sequence, neither purine/pyrimidine content nor distribution or total length of the ONs. To examine possible charge stabilization by larger counter ions, additionally single, double, triple, and quadruple sodium adducts of the 15-mers were investigated. However, no deviation of the characteristic fragmentation energies of the respective protonated species were observed. The fragmentation pattern and the parent ion stability appeared to depend both solely on the density of charges. The addition of charges always leads to a destabilization, except around the inflexion zone. A plausible reasoning for the observed behavior is a charge driven structural stabilization, which induces a conformational change that results in the observation of this plateau.

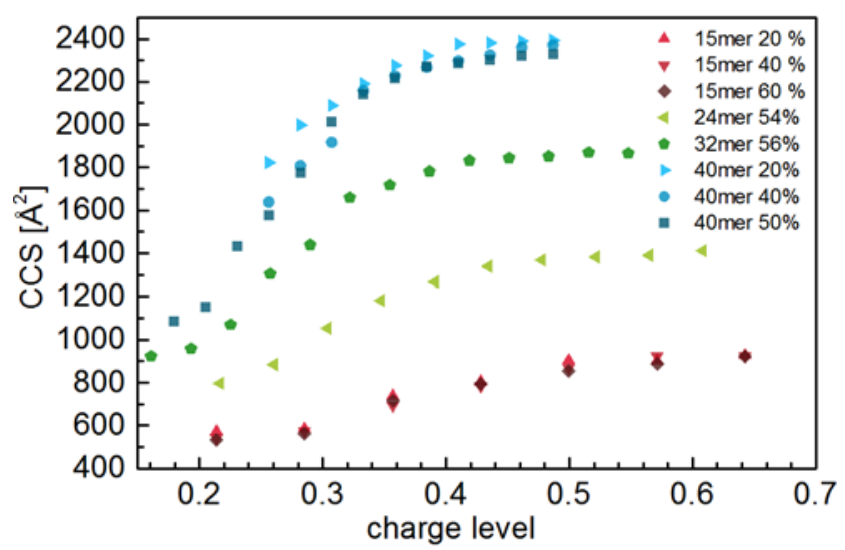

Figure 4. Correlation of CCSs to the negative charge / phosphate ratio of three 15-mer ONs (20, 40 and $60 \%$ GC content), three 40-mer ONs (20, 40 and $50 \%$ GC content), one 32-mer ON and one 24-mer ON.

To corroborate the hypothesis of a conformational change being responsible for the inflection region, ion mobility-mass spectrometry experiments were performed. In IM-MS ions traverse a cell, filled with an inert neutral gas, under the influence of a weak electric field, and are thus separated according to their mass, charge and shape. Compact ions undergo a small amount of collisions and have thus shorter drift times, while more extended structures interact more with the drift gas and have longer drift times. The observed drift times can be converted to rotationally-averaged CCSs, which are a molecular property that corresponds to the size and shape of ions. For ONs of each size, the CCSs of all observed charge states were determined in the drift gas helium. Fig. 4 shows a plot of CCSs against the charge level of the ions, which allows a comparison of the different ON sizes. The data shows in general an increase of CCSs with increasing number of charges. This is not surprising, because this coincides with an increase in Coulomb repulsion that results in an unfolding of ions. However, the unfolding seems to occur already at slightly smaller charge levels in comparison to the fragmentation points recorded on the ion trap. This deviation is most likely be caused by the different instruments and their setups. It was 
reported that additional heating of ions can occur in the used IM-MS device, which could be a reason for the observed experimental differences. ${ }^{[15]}$ Whether this increase in potential energy of the precursor ions originates from heating inside the ion source or is transferred to the ONs via inelastic collisions inside the drift tube remains unclear. ${ }^{[16,17]}$ Nevertheless, it can be clearly deducted that the relative trends of the IM-MS experiments and the fragmentation experiments on the ion trap are very similar. Along all populated charge states two linear zones exist, one at low and one at high charge levels. Between these zones a transition region can be found in all experiments. This similarity suggests the changes in fragmentation products and in dissociation energy correlate with a conformational change. It seems that with the addition of charges the fragmentation energy decreases until a further addition of charges leads to an unfolding of the molecule, which in turn stabilizes the molecule. As a result, for intermediate charge states similar collision energies are needed to fragment ions. Once the ion is unfolded, the increased size of the molecule and the larger number of charges again lead to a linear decrease of the necessary fragmentation energies. This dissociation trend might also be caused by different gas-phase conformations of the ONs. Different stabilization forces, like hydrogen bonds, zwitterionic or van der Waals forces influence structure as well as fragmentation behavior. ${ }^{[2,18]}$

The position of the inflexion zone in the fragmentation energy distributions fits in the generalized concept of charge density, since in all observed cases it was found around a charge level of about 0.5. At this charge level, every second phosphate carries a negative charge. An ON with few negative charges experiences less internal Coulomb repulsions in comparison to highly negatively charged molecules and is, therefore, able to maintain in a folded, more compact form. Whereas a highly charged ON needs to distribute the charges along a larger space, resulting in an elongated, linear structure.

\section{Conclusion}

The CID fragmentation product distribution, especially the ratio between neutral and negative base loss channels, strongly depends on the charge density. At low charge levels a strong propensity of the neutral base loss channel was observed until at a charge level of around 0.5 the negative base loss became the most abundant product channel. The characteristic fragmentation energies of all oligomers also followed a common trend. The energy necessary for the lowest dissociation channel decreased with increasing charge state. At low charge levels this decrease is linear, wheras at intermediate charge densities an inflexion zone occured beyond which the energy scaled linear with the charge level again. The slopes in the two distinctive linear regions appeared to be similar for the same oligonucleotide length. This observed trend was only dependent on the charge level of the respective ONs, while length, adduct formation and base identity did not have any influence. IM-MS studies of the ONs in different 
charge states revealed a conformational change upon increase in charge. Here, again a sudden increase in CCSs was observed at a defined charge level. This observation strongly suggests that with increasing charge state Coulomb repulsions increase, which lead to an unfolding of the molecule and thus lower fragmentation energies needed to fragment the precursor ions. At intermediate charge states this unfolding results in a stabilization of the molecule and thus a deviation from the linear behavior of the fragmentation energy destributions was observed. The results of the performed experiments clearly indicate that with increasing charge level gas-phase ONs undergo significant conformational changes that have a high influence on their fragmentation behavior.

\section{References}

[1] J. Wu, S. A. McLuckey, Int. J. Mass Spectrom. 2004, 237, 197-241.

[2] J. Gidden, M. T. Bowers, J. Am. Soc. Mass Spectrom. 2003, 14, 161-170.

[3] S. Pan, K. Verhoeven, J. K. Lee, J. Am. Soc. Mass Spectrom. 2005, 16, 1853-1865.

[4] S. A. McLuckey, G. Vaidyanathan, Int. J. Mass Spectrom. Ion Process. 1997, 162, 1-16.

[5] A. Favre, F. Gonnet, J. C. Tabet, Eur. J. Mass Spectrom. 2000, 6, 389-396.

[6] S. A. McLuckey, G. J. Van Berkel, D. E. Goeringer, G. L. Glish, Anal. Chem. 1994, 66, 737A743A.

[7] M. G. Bartlett, J. A. McCloskey, S. Manalili, R. H. Griffey, J. Mass Spectrom. 1996, 31, 12771283.

[8] F. Rosu, V. Gabelica, L. Joly, G. Gregoire, E. De Pauw, Phys. Chem. Chem. Phys. 2010, 12, 13448-13454.

[9] A. Arcella, J. Dreyer, E. Ippoliti, I. Ivani, G. Portella, V. Gabelica, P. Carloni, M. Orozco, Angew. Chem. Int. Ed. 2015, 54, 467-471.

[10] L. L. Lopez, P. R. Tiller, M. W. Senko, J. C. Schwartz, Rapid Commun. Mass Spectrom. 1999, 13, 663-668.

[11] S. J. Allen, K. Giles, T. Gilbert, M. F. Bush, Analyst 2016, 141, 884-891.

[12] H. E. Revercomb, E. A. Mason, Anal. Chem. 1975, 47, 970-983.

[13] G. von Helden, M. T. Hsu, N. Gotts, M. T. Bowers, J. Phys. Chem. 1993, 97, 8182-8192.

[14] S. A. McLuckey, S. Habibi-Goudarzi, J. Am. Soc. Mass Spectrom. 1994, 5, 740-747.

[15] D. Morsa, V. Gabelica, F. Rosu, J. Oomens, E. De Pauw, J. Phys. Chem. Lett. 2014, 5, 37873791.

[16] C. Larriba-Andaluz, C. J. Hogan, J. Chem. Phys. 2014, 141, 194107.

[17] W. F. Siems, L. A. Viehland, H. H. Hill, Anal. Chem. 2012, 84, 9782-9791.

[18] J. Abi-Ghanem, V. Gabelica, Phys. Chem. Chem. Phys. 2014, 16, 21204-21218. 